\title{
A Two-Dimensional Square Network Inclusion Compound Incorporating Guest Molecules Through Both Hydrogen Bonding and Nonionic Electrostatic Attraction. Crystal Structure of $\left[\mathrm{Cd}\left(4,4^{\prime}-\mathrm{bpy}\right)_{2}\left(\mathrm{H}_{2} \mathrm{O}\right)_{2}\right]-$ $\left(\mathrm{ClO}_{4}\right)_{2} \cdot 1.5\left(4,4^{\prime}-\right.$ bpy $) \cdot\left(\mathrm{C}_{6} \mathrm{H}_{4} \mathrm{NO}_{3} \mathrm{Cl}\right) \cdot \mathrm{H}_{2} \mathrm{O}$
}

\author{
Cai-Ming Liu, ${ }^{a}$ Ren-Gen Xiong, ${ }^{a}$ Xiao-Zeng You, ${ }^{*, a}$ and Wei Chen ${ }^{b}$
}

${ }^{a}$ Coordination Chemistry Institute, State Key Laboratory of Coordination Chemistry, Nanjing University, Center for

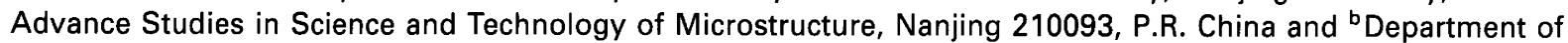
Chemistry, University of Malaya, Kuala Lumpur 59100, Malaysia

\begin{abstract}
Liu, C-M., Xiong, R-G., You, X-Z. and Chen, W., 1998. A Two-Dimensional Square Network Inclusion Compound Incorporating Guest Molecules Through Both Hydrogen Bonding and Nonionic Electrostatic Attraction. Crystal Structure of $\left[\mathrm{Cd}\left(4,4^{\prime} \text {-bpy }\right)_{2}\left(\mathrm{H}_{2} \mathrm{O}\right)_{2}\right]\left(\mathrm{ClO}_{4}\right)_{2} \cdot 1.5\left(4,4^{\prime}\right.$-bpy $) \cdot\left(\mathrm{C}_{6} \mathrm{H}_{4} \mathrm{NO}_{3} \mathrm{Cl}\right) \cdot \mathrm{H}_{2} \mathrm{O}$. - Acta Chem. Scand. 52: 1353-1358. (c) Acta Chemica Scandinavica 1998.

The two-dimensional square network inclusion compound $\left[\mathrm{Cd}\left(4,4^{\prime}-\text {-bpy }\right)_{2}\left(\mathrm{H}_{2} \mathrm{O}\right)_{2}\right]-$ $\left(\mathrm{ClO}_{4}\right)_{2} \cdot 1.5\left(4,4^{\prime}\right.$-bpy) $\cdot\left(\mathrm{C}_{6} \mathrm{H}_{4} \mathrm{NO}_{3} \mathrm{Cl}\right) \cdot \mathrm{H}_{2} \mathrm{O}$ (where $\mathrm{C}_{6} \mathrm{H}_{4} \mathrm{NO}_{3} \mathrm{Cl}=4$-chloro-2nitrophenol, $4,4^{\prime}$-bpy $=4,4^{\prime}$-bipyridine) has been synthesized by self-assembly. The compound crystallizes in the monoclinic space group $P 2$ with unit-cell parameters $a=11.7365(8), b=11.7808(7), c=16.851(1) \AA, \beta=93.35(4)^{\circ}, V=$ $2327.9(3) \AA^{3}, Z=4, R_{1}=0.0351$ and $w R_{2}=0.0813$. The central $C d$ atoms in two crystallographically non-equivalent $\left[\mathrm{Cd}\left(4,4^{\prime}-\text { bpy }\right)_{2}\left(\mathrm{H}_{2} \mathrm{O}\right)_{2}\right]^{2+}$ cations exhibit a slightly distorted octahedral coordination geometry with $\mathrm{Cd}-\mathrm{N}$ bond distances of 2.329(5)-2.382(11) and 2.324(10)-2.364(5) $\AA$, respectively, and $\mathrm{Cd}-\mathrm{O}$ bond distances of 2.315(4) and 2.324(5) A, respectively. Both hydrogen bonding and nonionic electrostatic attraction are utilized to incorporate the 4-chloro-2nitrophenol guest molecules, thus making the self-assembly process exhibit high shape specificity. The thermal analysis of the compound is discussed and related to its structure.
\end{abstract}

\begin{abstract}
There is current interest in crystal engineering of highdimensional coordination polymers due to both their potential physicochemical properties and their theoretical aspects related to topologies of novel networks. ${ }^{1-5}$ Furthermore, since very specific pore size, type and functions of cavity become designable, their applications in inclusion chemistry are particularly attractive. ${ }^{6}$ Fujita et $a l^{7}$ have recently reported the clathration ability of a two-dimensional (2D) square network material $\left\{\left[\mathrm{Cd}\left(4,4^{\prime} \text {-bpy }\right)_{2}\right]\left(\mathrm{NO}_{3}\right)_{2}\right\}_{\infty} \quad\left(4,4^{\prime}\right.$-bpy $=4,4^{\prime}$-bipyridine $)$ and described the crystal structure of $\left[\mathrm{Cd}\left(4,4^{\prime}\right.\right.$ bpy $\left.)_{2}\right]\left(\mathrm{NO}_{3}\right)_{2} \cdot\left(\mathrm{C}_{6} \mathrm{H}_{4} \mathrm{Br}_{2}\right)_{2}$ in which nonionic electrostatic attraction is utilized to incorporate $o$-dibromobenzene guest molecules. However, 2D rigid square network inclusion compounds which incorporate guest molecules through hydrogen bonds are relatively unexplored. Here we report the molecular recognition of nitrophenol derivatives using a 2D square network of $\left[\mathrm{Cd}\left(4,4^{\prime}-\mathrm{bpy}\right)_{2}\left(\mathrm{H}_{2} \mathrm{O}\right)_{2}\right]^{2+}$ ions and tetrahedral anions
\end{abstract}

* To whom correspondence should be addressed.
$\left(\mathrm{ClO}_{4}{ }^{-}\right)$and the crystal structure of $\left[\mathrm{Cd}\left(4,4^{\prime}\right.\right.$ bpy $\left.)_{2}\left(\mathrm{H}_{2} \mathrm{O}\right)_{2}\right]\left(\mathrm{ClO}_{4}\right)_{2} \cdot 1.5\left(4,4^{\prime}\right.$-bpy $) \cdot\left(\mathrm{C}_{6} \mathrm{H}_{4} \mathrm{NO}_{3} \mathrm{Cl}\right) \cdot$ $\mathrm{H}_{2} \mathrm{O}$, where 4-chloro-2-nitrophenol guest molecules are incorporated by both hydrogen bonding and nonionic electrostatic attraction.

\section{Experimental}

All materials were reagent grade and were used without further purification. Doubly distilled water was used throughout this work. Caution: Care must be taken when perchlorate salts are used as they sometimes explode.

$\left[\mathrm{Cd}\left(4,4^{\prime} \text {-bpy }\right)_{2}\left(\mathrm{H}_{2} \mathrm{O}\right)_{2}\right]\left(\mathrm{ClO}_{4}\right)_{2} \cdot 1.5\left(4,4^{\prime}\right.$-bpy $)$. $\left(\mathrm{C}_{6} \mathrm{H}_{4} \mathrm{NO}_{3} \mathrm{Cl}\right) \cdot \mathrm{H}_{2} \mathrm{O}$ was prepared by dissolving $\mathrm{Cd}\left(\mathrm{ClO}_{4}\right)_{2} \cdot 6 \mathrm{H}_{2} \mathrm{O}(1.0 \mathrm{mmol})$ in $15 \mathrm{~cm}^{3}$ of methanol and water ( V/V 1:1) and adding this solution to a solution of the 4,4'-bpy ligand $(4.0 \mathrm{mmol})$ and 4-chloro-2-nitrophenol $(1.5 \mathrm{mmol})$ in $20 \mathrm{~cm}^{3}$ of methanol. The solution was refluxed for $1 \mathrm{~h}$, after the solution was cooled to room temperature, the filtrate was then left to evaporate after a few weeks to give the yellow block crystals. The 
products were collected by filtration, washed with methanol, and dried in air. Yield $70-80 \%$. Anal. Calcd for $\left[\mathrm{Cd}\left(4,4^{\prime} \text {-bpy }\right)_{2}\left(\mathrm{H}_{2} \mathrm{O}\right)_{2}\right]\left(\mathrm{ClO}_{4}\right)_{2} \cdot 1.5\left(4,4^{\prime}\right.$-bpy $) \cdot$ $\left(\mathrm{C}_{6} \mathrm{H}_{4} \mathrm{NO}_{3} \mathrm{Cl}\right) \cdot \mathrm{H}_{2} \mathrm{O}: \mathrm{C}, 45.4 ; \mathrm{H}, 3.5 ; \mathrm{N}, 10.3$; Found: $\mathrm{C}, 45.2 ; \mathrm{H}, 3.6 ; \mathrm{N}, 10.2 \%$. IR ( $\mathrm{cm}^{-1}, \mathrm{KBr}$ discs, Nujol $)$ : 3407(m), 3385(m), 3206(m), 3095(m), 3055(m), 1602(s), 1535(m), 1515(m), 1490(w), 1474(w), 1412(s), 1346(w), $1317(\mathrm{w}), 1261(\mathrm{w}), 1220(\mathrm{~m}), 1108(\mathrm{~s}), 1094(\mathrm{~s}), 1008(\mathrm{~m})$, $860(w), 810(s), 628(s)$.

Physical measurements. Elemental analyses for C, H, N were performed on a Perkin-Elmer 240 elemental analyzer. IR spectrum was recorded by aid of $\mathrm{KBr}$ pellets in the $4000-400 \mathrm{~cm}^{-1}$ in Nujol mull modes using a Nicolet 170 SXFTIR spectrophotometer. Thermogravimetric and differential thermal analyses were performed on a Simultanous SDT 2960 thermal analyzer under flowing $\mathrm{N}_{2}$ with a heating rate of $10^{\circ} \mathrm{C} \min ^{-1}$.

Crystal structure determination. A yellow block crystal of approximate dimensions $0.36 \times 0.3 \times 0.3 \mathrm{~mm}$ was coated with epoxy glue and mounted on the tip of a glass fiber. Data were collected at room temperature on an Enraf-Nonius CAD4 diffractometer with graphitemonochromated Mo-K $\alpha$ radiation $(\lambda=0.71073 \AA)$ using the $\omega-2 \theta$ scan technique. Cell constraints and the orientation matrix for intensity collection were based on the setting angles of 25 carefully centered reflections with $2 \theta$ values between 9 and $22^{\circ}$. A total of 4548 reflections were collected, of which 4343 independent reflections $\left(R_{\text {int }}=0.0273\right)$ and 3862 reflections observed with $I>2 \sigma(I)$ were used in the structure solution and refinement. Data were corrected for Lorentz and polarization effects, and an empirical absorption based on $\psi$-scan was applied. A summary of crystal data collection parameters are listed in Table 1.

Structure solution and refinement. The structure was solved by Patterson methods using SHELXS 86 program $^{8}$ and expanded by Fourier techniques assuming the monoclinic space group $P 2$. A total of 609 parameters with non-hydrogen atoms assumed to vibrate anisotropically were refined by the full-matrix least-squares on $F_{\mathrm{o}}{ }^{2}$, where $\omega=1 /\left[\sigma^{2}\left(F_{\mathrm{o}}{ }^{2}\right)+(0.0448 P)^{2}+1.3771 P\right)$ and $P=$ $\left(F_{\mathrm{o}}{ }^{2}+2 F_{\mathrm{c}}{ }^{2}\right) / 3$. Refinement converged at $R_{1}=0.0351$, ${ }_{\mathrm{w}} R_{2}=0.0813 \quad\left[I>2 \sigma(I)\right.$ observed data]; $R_{1}=0.0419$, ${ }_{\mathrm{w}} R_{2}=0.0843$ (all data). All hydrogen atoms on the 4,4 ' bpy ligands and the benzene ring of the 4-chloride-2nitrophenol guest molecules were generated geometrically and given a riding mode with $U=1.2$ times that of the parent carbon atoms. The hydrogen atoms on water and $\mathrm{OH}$ were located from difference maps but not refined. The final difference electron density map showed some residual electron density $\left(-0.310 \leqslant \Delta \rho \leqslant 0.509\right.$ e $\left.\AA^{-3}\right)$. The refinement program used is SHELXL 93. ${ }^{9}$
Table 1. Summary of crystal data of the title compound.

\begin{tabular}{ll}
\hline Empirical formula & $\mathrm{C}_{41} \mathrm{H}_{38} \mathrm{~N}_{8} \mathrm{O}_{14} \mathrm{Cl}_{3} \mathrm{Cd}$ \\
$M_{\mathrm{r}}$ & 1085.54 \\
Crystal system & Monoclinic \\
Space group & $P 2$ \\
$T / \mathrm{K}$ & $300(2)$ \\
$a / \AA$ & $11.7365(8)$ \\
$b / \AA$ & $11.7808(7)$ \\
$c / \AA$ & $16.851(1)$ \\
$\beta /{ }^{\circ}$ & $92.386(6)$ \\
$V / \AA^{3}$ & $2327.9(3)$ \\
$Z$ & 2 \\
$D_{\text {call }} / \mathrm{g} \mathrm{cm}^{-3}$ & 1.549 \\
$\mu\left(M_{\mathrm{o}} \mathrm{K}_{\alpha}\right) / \mathrm{cm}^{-1}$ & 7.15 \\
$F(000)$ & 1102 \\
$\theta-$ Range collected $/$ & $1.21-25.06$ \\
No. of reflections collected & 4548 \\
No. of independent reflections & $4343\left(R_{\text {int }}=0.0273\right)$ \\
No. of observed data $[I>2 \sigma(I)]$ & 3862 \\
Data/restraints/parameters & $4343 / 1 / 606$ \\
Final $R[I>2 \sigma(I)]$ & $R_{1}=0.0351,{ }_{w} R_{2}=0.0813$ \\
$R$-indices (all data) & $R_{1}=0.0419,{ }_{w} R_{2}=0.0843$ \\
GOF $[I>2 \sigma(I)]^{a}$ & 1.099 \\
GOF (all data) & 1.065 \\
$T_{\text {max }} / T_{\text {min }}$ & $0.807 / 0.765$ \\
\hline
\end{tabular}

${ }^{a}$ GOF, Goodness of fit.

\section{Results and discussion}

Preparation. High shape specificity in the clathration was obtained during the self-assembly process owing to 4-chloro-2-nitrophenol guest molecules in $\left[\mathrm{Cd}\left(4,4^{\prime}\right.\right.$ bpy $\left.)_{2}\left(\mathrm{H}_{2} \mathrm{O}\right)_{2}\right]\left(\mathrm{ClO}_{4}\right)_{2} \cdot 1.5\left(4,4^{\prime}\right.$-bpy $) \cdot\left(\mathrm{C}_{6} \mathrm{H}_{4} \mathrm{NO}_{3} \mathrm{Cl}\right) \cdot$ $\mathrm{H}_{2} \mathrm{O}$ being incorporated by both hydrogen bonding and nonionic electrostatic attraction, as indicated in the crystal structure below. Whereas 4-chloro-2-nitrophenol was included efficiently, 4-chlorophenol, 2-nitrophenol and the 4-chloro-2-nitrophenol's isomers (5-chloro-2-nitrophenol and 2-chloro-4-nitrophenol) were not included. This discrimination of the isomers in the clathration could be utilized to separate the 4-chloro-2-nitrophenol from the isomer mixtures as previously reported. ${ }^{10}$ Thus treatment of a mixture of 4-chloro-2-nitrophenol, 5chloro-2-nitrophenol and 2-chloro-4-nitrophenol (1:1:1) with $\mathrm{Cd}\left(\mathrm{ClO}_{4}\right)_{2} \cdot 6 \mathrm{H}_{2} \mathrm{O}$ and $4,4^{\prime}$-bpy in methanol-water yielded only $\left[\mathrm{Cd}\left(4,4^{\prime}-\text { bpy }\right)_{2}\left(\mathrm{H}_{2} \mathrm{O}\right)_{2}\right]\left(\mathrm{ClO}_{4}\right)_{2} \cdot 1.5\left(4,4^{\prime}\right.$ bpy) $\cdot\left(\mathrm{C}_{6} \mathrm{H}_{4} \mathrm{NO}_{3} \mathrm{Cl}\right) \cdot \mathrm{H}_{2} \mathrm{O}$ as inclusion crystals, from which the 4-chloro-2-nitrophenol could be recovered by an acidic decomposition of $\left[\mathrm{Cd}\left(4,4^{\prime}\right.\right.$ bpy $\left.)_{2}\left(\mathrm{H}_{2} \mathrm{O}\right)_{2}\right]\left(\mathrm{ClO}_{4}\right)_{2} \cdot 1.5\left(4,4^{\prime}\right.$-bpy $) \cdot\left(\mathrm{C}_{6} \mathrm{H}_{4} \mathrm{NO}_{3} \mathrm{Cl}\right) \cdot$ $\mathrm{H}_{2} \mathrm{O}$ followed by recrystallization.

Crystal structure of $\left[\mathrm{Cd}\left(4,4^{\prime}-b p y\right)_{2}\left(\mathrm{H}_{2} \mathrm{O}\right)_{2}\right]\left(\mathrm{ClO}_{4}\right)_{2}$. $1.5\left(4,4^{\prime}-b p y\right) \cdot\left(\mathrm{C}_{6} \mathrm{H}_{4} \mathrm{NO}_{3} \mathrm{Cl}\right) \cdot \mathrm{H}_{2} \mathrm{O}$. An ORTEP drawing of the structure around the cadmium atoms in $\left[\mathrm{Cd}\left(4,4^{\prime}-\right.\right.$ bpy $\left.)_{2}\left(\mathrm{H}_{2} \mathrm{O}\right)_{2}\right]\left(\mathrm{ClO}_{4}\right)_{2} \cdot 1.5\left(4,4^{\prime}\right.$-bpy $) \cdot\left(\mathrm{C}_{6} \mathrm{H}_{4} \mathrm{NO}_{3} \mathrm{Cl}\right) \cdot$ $\mathrm{H}_{2} \mathrm{O}$ with the atom numbering scheme is shown in Fig. 1 . Atomic coordinates for non-hydrogen atoms of the title compound are given in Table 2. Selected bond distances and angles are listed in Table 3. The structure of $\left[\mathrm{Cd}\left(4,4^{\prime}-\right.\right.$ bpy $\left.)_{2}\left(\mathrm{H}_{2} \mathrm{O}\right)_{2}\right]\left(\mathrm{ClO}_{4}\right)_{2} \cdot 1.5\left(4,4^{\prime}\right.$-bpy $) \cdot\left(\mathrm{C}_{6} \mathrm{H}_{4} \mathrm{NO}_{3} \mathrm{Cl}\right) \cdot$ 


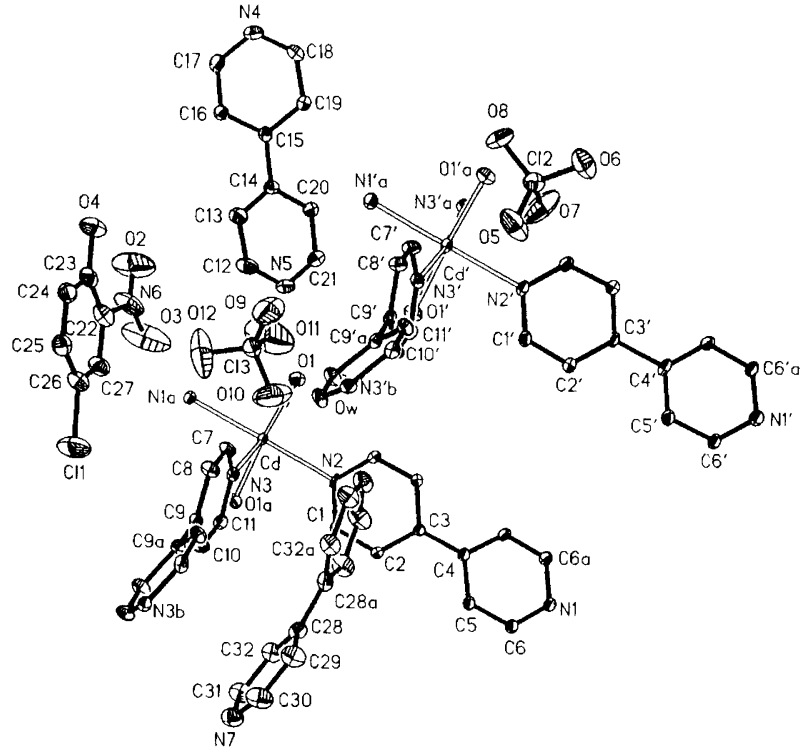

Fig. 1. The structure around the cadmium atoms of the title compound with atomic numbering.
$\mathrm{H}_{2} \mathrm{O}$ shows two crystallographically independent $\left[\mathrm{Cd}\left(4,4^{\prime} \text {-bpy }\right)_{2}\left(\mathrm{H}_{2} \mathrm{O}\right)_{2}\right]^{2+}$ cations and two crystallographically independent uncoordinated 4,4'-bpy molecules. The cadmium atoms are situated on two-fold axes and one uncoordinated 4,4'-bpy molecule lies across the two-fold rotation axis. The cadmium atoms exhibit a slightly distorted octahedral geometry in which two trans coordinated water molecules occupy the apical positions and four pyridyl nitrogen donors the equatorial positions. This local coordinate environment is very similar to that in $\left[\mathrm{Cd}\left(4,4^{\prime} \text {-bpy }\right)_{2}\left(\mathrm{H}_{2} \mathrm{O}\right)_{2}\right]\left(\mathrm{PF}_{6}\right)_{2} \cdot 2\left(4,4^{\prime}-\right.$ bpy $) \cdot 4 \mathrm{H}_{2} \mathrm{O}^{1 \mathrm{a}}$ but slightly different from that in $\left[\mathrm{Cd}\left(4,4^{\prime}\right.\right.$ bpy $\left.)_{2}\left(\mathrm{NO}_{2}\right)_{2}\right] \cdot\left(\mathrm{C}_{6} \mathrm{H}_{4} \mathrm{Br}_{2}\right)_{2}$, where four pyridyl groups occupy the equatorial and two nitrate ions the apical positions. ${ }^{7}$ The bond distances of $\mathrm{Cd}-\mathrm{O}_{\mathrm{w}}$ [average 2.315(4) and 2.324(5) $\AA$ for $\mathrm{Cd}$ and $\mathrm{Cd}^{\prime}$, respectively] and $\mathrm{Cd}-\mathrm{N}$ (average 2.358, $2.341 \AA$ for $\mathrm{Cd}, \mathrm{Cd}^{\prime}$, respectively) are comparable with those in $\left[\mathrm{Cd}\left(4,4^{\prime}\right.\right.$ bpy $\left.)_{2}\left(\mathrm{H}_{2} \mathrm{O}\right)_{2}\right]\left(\mathrm{PF}_{6}\right)_{2} \cdot 2\left(4,4^{\prime}\right.$-bpy $) \cdot 4 \mathrm{H}_{2} \mathrm{O} .{ }^{1 \text { a }}$ The coordinated 4,4'-bpy ligands are not coplanar: the dihedral angles between the pyridyl rings in each coordinated bipyridine molecule are $47.1(3)$ and $36.4(2)^{\circ}$ for the

Table 2. Atomic coordinates (in $\AA$ ) and equivalent isotropic displacement parameters $\left(\right.$ in $\AA^{2}$ ) for the title complex.

\begin{tabular}{|c|c|c|c|c|c|c|c|c|c|}
\hline Atom & $x / a$ & $y / b$ & $z / c$ & $U_{\text {iso/eq }}{ }^{a}$ & Atom & $x / a$ & $y / b$ & $z / c$ & $U_{\text {iso/eq }}{ }^{a}$ \\
\hline $\mathrm{Cd}$ & 0.0000 & $0.1729(1)$ & 0.5000 & $0.026(1)$ & $C(7)$ & $0.2542(5)$ & $0.2156(7)$ & $0.4373(4)$ & $0.043(2)$ \\
\hline $\mathrm{Cd}^{\prime}$ & 0.0000 & $-0.0002(1)$ & 0.0000 & $0.028(1)$ & $C(8)$ & $0.3692(5)$ & $0.2211(7)$ & $0.4342(4)$ & $0.044(2)$ \\
\hline $\mathrm{Cl}(1)$ & $0.6030(3)$ & $0.4865(4)$ & $0.4146(2)$ & $0.130(1)$ & $C(9)$ & $0.4378(5)$ & $0.1928(6)$ & $0.5005(4)$ & $0.035(2)$ \\
\hline $\mathrm{Cl}(2)$ & $0.2537(2)$ & $-0.1099(2)$ & $-0.2478(1)$ & $0.058(1)$ & $C(10)$ & $0.3813(6)$ & $0.1606(8)$ & $0.5682(4)$ & $0.042(2)$ \\
\hline $\mathrm{Cl}(3)$ & $0.3752(2)$ & $0.2597(2)$ & $0.1921(1)$ & $0.070(1)$ & $C(11)$ & $0.2628(5)$ & $0.1552(6)$ & $0.5649(4)$ & $0.038(2)$ \\
\hline$O(1)$ & $-0.0057(4)$ & $0.1653(6)$ & $0.3626(3)$ & $0.042(1)$ & $C\left(1^{\prime}\right)$ & $0.0457(7)$ & $-0.2583(7)$ & $0.0605(5)$ & $0.044(2)$ \\
\hline$O\left(1^{\prime}\right)$ & $-0.0005(4)$ & $-0.0066(7)$ & $0.1378(3)$ & $0.050(2)$ & $C\left(2^{\prime}\right)$ & $0.0475(7)$ & $-0.3764(7)$ & $0.0631(5)$ & $0.042(2)$ \\
\hline$O(2)$ & $0.1263(14)$ & $0.6343(17)$ & $0.2815(10)$ & $0.116(6)$ & $C\left(3^{\prime}\right)$ & 0.0000 & $-0.4357(10)$ & 0.0000 & $0.034(3)$ \\
\hline$O\left(2^{*}\right)$ & $0.1390(19)$ & $0.5715(24)$ & $0.2502(15)$ & $0.103(9)$ & $C\left(4^{\prime}\right)$ & 0.0000 & $-0.5660(10)$ & 0.0000 & $0.040(3)$ \\
\hline$O(3)$ & $0.1712(10)$ & $0.4836(13)$ & $0.3584(7)$ & $0.090(5)$ & $C\left(5^{\prime}\right)$ & $0.0707(8)$ & $-0.6252(8)$ & $0.0507(5)$ & $0.049(2)$ \\
\hline$O\left(3^{*}\right)$ & $0.1717(22)$ & $0.5762(28)$ & $0.3700(17)$ & $0.125(11)$ & $C\left(6^{\prime}\right)$ & $0.0676(7)$ & $-0.7416(8)$ & $0.0499(5)$ & $0.047(2)$ \\
\hline$O(4)$ & $0.2840(5)$ & $0.7027(7)$ & $0.1827(4)$ & $0.089(2)$ & $C\left(7^{\prime}\right)$ & $0.2516(5)$ & $0.0556(6)$ & $-0.0543(4)$ & $0.041(2)$ \\
\hline $\mathrm{O}(5)$ & $0.3597(6)$ & $-0.1165(9)$ & $-0.2067(5)$ & $0.125(3)$ & $C\left(8^{\prime}\right)$ & $0.3685(5)$ & $0.0587(6)$ & $-0.0601(4)$ & $0.042(2)$ \\
\hline$O(6)$ & $0.2570(6)$ & $-0.1833(8)$ & $-0.3138(4)$ & $0.105(3)$ & $C\left(9^{\prime}\right)$ & $0.4362(5)$ & $0.0185(6)$ & $0.0020(4)$ & $0.029(2)$ \\
\hline$O(7)$ & $0.1735(9)$ & $-0.1484(8)$ & $-0.1942(6)$ & $0.151(4)$ & $C\left(10^{\prime}\right)$ & $0.3836(5)$ & $-0.0245(7)$ & $0.0671(4)$ & $0.033(2)$ \\
\hline$O(8)$ & $0.2195(10)$ & $-0.0020(8)$ & $-0.2705(5)$ & $0.149(4)$ & $C\left(11^{\prime}\right)$ & $0.2676(5)$ & $-0.0271(6)$ & $0.0684(4)$ & $0.035(2)$ \\
\hline$O(9)$ & $0.3940(13)$ & $0.2613(9)$ & $0.1133(5)$ & $0.183(6)$ & $C(12)$ & $-0.0979(7)$ & $0.4015(9)$ & $0.2545(5)$ & $0.069(2)$ \\
\hline$O(10)$ & $0.4508(13)$ & $0.1706(13)$ & $0.2257(7)$ & $0.108(6)$ & $C(13)$ & $-0.1450(6)$ & $0.4754(7)$ & $0.1984(4)$ & $0.054(2)$ \\
\hline$O\left(10^{*}\right)$ & $0.3553(37)$ & $0.1476(36)$ & $0.2227(20)$ & $0.184(16)$ & $C(14)$ & $-0.2406(6)$ & $0.4452(6)$ & $0.1548(4)$ & $0.042(2)$ \\
\hline $\mathrm{O}(11)$ & $0.2674(17)$ & $0.2293(21)$ & $0.1964(11)$ & $0.149(8)$ & $C(15)$ & $-0.2873(5)$ & $0.5185(6)$ & $0.0899(4)$ & $0.043(2)$ \\
\hline$O\left(11^{*}\right)$ & $0.2696(32)$ & $0.3209(40)$ & $0.2150(21)$ & $0.170(16)$ & $C(16)$ & $-0.2804(7)$ & $0.6355(6)$ & $0.0941(4)$ & $0.051(2)$ \\
\hline$O(12)$ & $0.4114(16)$ & $0.3620(19)$ & $0.2208(11)$ & $0.142(7)$ & $C(17)$ & $-0.3158(7)$ & $0.6986(7)$ & $0.0303(4)$ & $0.059(2)$ \\
\hline$O\left(12^{*}\right)$ & $0.4554(26)$ & $0.3040(28)$ & $0.2489(17)$ & $0.132(11)$ & $C(18)$ & $-0.3668(7)$ & $0.5443(8)$ & $-0.0413(4)$ & $0.064(2)$ \\
\hline$N(1)$ & 0.0000 & $-0.6249(9)$ & 0.5000 & $0.037(3)$ & $C(19)$ & $-0.3349(6)$ & $0.4723(6)$ & $0.0200(4)$ & $0.051(2)$ \\
\hline$N\left(1^{\prime}\right)$ & 0.0000 & $-0.8020(9)$ & 0.0000 & $0.042(3)$ & $C(20)$ & $-0.2890(7)$ & $0.3413(7)$ & $0.1720(5)$ & $0.059(2)$ \\
\hline$N(2)$ & 0.0000 & $-0.0277(7)$ & 0.5000 & $0.035(2)$ & $C(21)$ & $-0.2370(9)$ & $0.2728(8)$ & $0.2290(6)$ & $0.076(3)$ \\
\hline$N\left(2^{\prime}\right)$ & 0.0000 & $-0.1975(8)$ & 0.0000 & $0.035(3)$ & $C(22)$ & $0.3192(7)$ & $0.5947(9)$ & $0.3012(5)$ & $0.068(2)$ \\
\hline$N(3)$ & $0.1983(4)$ & $0.1825(7)$ & $0.5013(3)$ & $0.032(1)$ & $C(23)$ & $0.3567(8)$ & $0.6542(7)$ & $0.2376(5)$ & $0.063(2)$ \\
\hline$N\left(3^{\prime}\right)$ & $0.2011(4)$ & $0.131(7)$ & $0.080(3)$ & $0.033(1)$ & $C(24)$ & $0.4727(7)$ & $0.6610(8)$ & $0.2307(5)$ & $0.065(2)$ \\
\hline$N(4)$ & $-0.3572(6)$ & $0.6563(7)$ & $-0.0374(4)$ & $0.068(2)$ & $C(25)$ & $0.5484(7)$ & $0.6122(8)$ & $0.2845(5)$ & $0.067(2)$ \\
\hline$N(5)$ & $-0.1438(6)$ & $0.3001(6)$ & $0.2702(4)$ & $0.064(2)$ & $C(26)$ & $0.5071(7)$ & $0.5537(9)$ & $0.3482(5)$ & $0.066(2)$ \\
\hline$N(6)$ & $0.1968(8)$ & $0.5713(13)$ & $0.3115(6)$ & $0.116(4)$ & $C(27)$ & $0.3929(8)$ & $0.5440(9)$ & $0.3565(5)$ & $0.070(2)$ \\
\hline$N(7)$ & $0.7106(6)$ & $-0.0961(7)$ & $0.6554(5)$ & $0.069(2)$ & $C(28)$ & $0.5430(6)$ & $-0.1275(6)$ & $0.5332(4)$ & $0.050(2)$ \\
\hline$C(1)$ & $0.0431(7)$ & $-0.0850(7)$ & $0.5628\langle 4\rangle$ & $0.038(2)$ & $C(29)$ & $0.6573(7)$ & $-0.1465(9)$ & $0.5209(5)$ & $0.074(3)$ \\
\hline$C(2)$ & $0.0459(7)$ & $-0.2029(7)$ & $0.5644(5)$ & $0.039(2)$ & $C(30)$ & $0.7350(7)$ & $-0.1268(10)$ & $0.5822(6)$ & $0.080(3)$ \\
\hline$C(3)$ & 0.0000 & $-0.2651(10)$ & 0.5000 & $0.030(3)$ & $C(31)$ & $0.5993(7)$ & $-0.0831(9)$ & $0.6674(5)$ & $0.069(2)$ \\
\hline$C(4)$ & 0.0000 & $-0.3875(11)$ & 0.5000 & $0.035(3)$ & $C(32)$ & $0.5165(7)$ & $-0.0992(8)$ & $0.6096(5)$ & $0.063(2)$ \\
\hline$C(5)$ & $0.0845(6)$ & $-0.4517(6)$ & $0.5392(5)$ & $0.039(2)$ & $\mathrm{O}_{\mathrm{w}}$ & $0.1440(4)$ & $0.0538(6)$ & $0.2614(3)$ & $0.066(2)$ \\
\hline$C(6)$ & $0.0832(6)$ & $-0.5677(7)$ & $0.5382(5)$ & $0.039(2)$ & & & & & \\
\hline
\end{tabular}

${ }^{a} U_{\text {iso/eq }}$ is defined as one third of the trace of the orthogonalized $U_{\mathrm{ij}}$ tensor. 
Table 3. Selected bond lengths (in $\AA$ ) and bond angles (in ${ }^{\circ}$ ) of the title compound.

\begin{tabular}{|c|c|c|c|}
\hline \multicolumn{4}{|l|}{ Bond lengths $/ \AA$} \\
\hline $\mathrm{Cd}-\mathrm{O}(1)$ & $\begin{array}{l}2.315(4) \\
2.329(5)\end{array}$ & $C d^{\prime}-O(1)^{\prime}$ & $2.324(5)$ \\
\hline $\mathrm{Cd}-\mathrm{N}(3)$ & $2.329(5)$ & $\mathrm{Cd}-\mathrm{N}(1)^{\prime \prime 1}$ & $2.336(11)$ \\
\hline $\mathrm{Cd}-\mathrm{N}(2)$ & $2.363(8)$ & $C d^{\prime}-N(2)^{\prime}$ & $2.324(10)$ \\
\hline $\mathrm{Cd}-\mathrm{N}(1)^{\# 1}$ & $2.382(11)$ & $\mathrm{Cd}^{\prime}-\mathrm{N}(3)^{\prime}$ & $2.364(5)$ \\
\hline$N(1)-C(6)$ & $1.330(9)$ & $N(1)^{\prime}-C(6)^{\prime}$ & $1.337(10)$ \\
\hline$N(2)-C(1)$ & $1.337(9)$ & $\mathrm{N}(2)^{\prime}-\mathrm{C}(1)^{\prime}$ & $1.340(10)$ \\
\hline$N(3)-C(7)$ & $1.343(8)$ & $\mathrm{N}(3)^{\prime}-\mathrm{C}(7)^{\prime}$ & $1.325(8)$ \\
\hline$N(3)-C(11)$ & $1.326(8)$ & $\mathrm{N}(3)^{\prime}-\mathrm{C}(11)^{\prime}$ & $1.342(8)$ \\
\hline$N(4)-C(18)$ & $1.319(10)$ & $N(6)-C(22)$ & $1.479(12)$ \\
\hline$N(4)-C(17)$ & $1.319(10)$ & $N(7)-C(31)$ & $1.333(10)$ \\
\hline$N(5)-C(12)$ & $1.323(11)$ & $N(7)-C(30)$ & $1.327(12)$ \\
\hline$N(5)-C(21)$ & $1.312(11)$ & $\mathrm{O}(2)-\mathrm{N}(6)$ & $1.21(2)$ \\
\hline $\mathrm{O}(3)-\mathrm{N}(6)$ & $1.34(2)$ & $\mathrm{O}(4)-\mathrm{C}(23)$ & $1.358(10)$ \\
\hline$C(3)-C(4)$ & $1.44(2)$ & $C(3)^{\prime}-C(4)^{\prime}$ & $1.54(2)$ \\
\hline$C(9)-C(9)^{\# 2}$ & $1.460(12)$ & $C(9)^{\prime}-C(9)^{\prime \prime 3}$ & $1.501(11)$ \\
\hline$C(14)-C(15)$ & $1.479(9)$ & $C(28)-C(28)^{\# 2}$ & $1.474(14)$ \\
\hline$O(4)-C(23)$ & $1.358(10)$ & $\mathrm{Cl}(1)-\mathrm{C}(26)$ & $1.744(9)$ \\
\hline \multicolumn{4}{|l|}{ Bond angles/ ${ }^{\circ}$} \\
\hline$O(1)^{* 4}-C d-O(1)$ & $175.5(4)$ & $\mathrm{O}(1)^{\prime \prime * 5}-\mathrm{Cd}^{\prime}-\mathrm{O}(1)^{\prime}$ & $176.3(4)$ \\
\hline $\mathrm{O}(1)-\mathrm{Cd}-\mathrm{N}(3)^{\# 4}$ & $90.3(2)$ & $O(1)^{\prime}-C d^{\prime}-N(1)^{\prime * 11}$ & $91.8(2)$ \\
\hline$O(1)-C d-N(3)$ & $89.9(2)$ & $O(1)^{\prime}-C d^{\prime}-N(3)^{\prime}$ & $89.4(2)$ \\
\hline$N(3)^{\# 4}-C d-N(3)$ & $174.4(4)$ & $N(1)^{\prime * 1}-C d^{\prime}-N(2)^{\prime}$ & 180.0 \\
\hline $\mathrm{O}(1)-\mathrm{Cd}-\mathrm{N}(2)$ & $87.8(2)$ & $O(1)^{\prime}-C d^{\prime}-N(3)^{\prime * 5}$ & $90.9(2)$ \\
\hline$N(3)-C d-N(2)$ & $92.8(2)$ & $O(1)^{\prime}-C d^{\prime}-N(3)^{\prime}$ & $89.4(2)$ \\
\hline$O(1)-C d-N(1)^{\# 1}$ & $92.2(2)$ & $N(1)^{\# 1}-C d^{\prime}-N(3)^{\prime}$ & $86.2(2)$ \\
\hline$N(3)-C d-N(1)^{\# 1}$ & $87.2(2)$ & $N(2)^{\prime}-C d^{\prime}-N(3)^{\prime}$ & $93.8(2)$ \\
\hline $\mathrm{N}(2)-\mathrm{Cd}-\mathrm{N}(1)^{\# 1}$ & $180.000(2)$ & $N(3)^{\prime \prime 45}-C d^{\prime}-N(3)^{\prime}$ & $172.4(4)$ \\
\hline$C(6)-N(1)-C d^{\# 6}$ & $120.4(5)$ & $C(6)^{\prime}-N(1)^{\prime}-C^{\prime \prime \prime 6}$ & $122.1(6)$ \\
\hline$C(1)-N(2)-C d$ & $120.3(5)$ & $\mathrm{C}(1)^{\prime}-\mathrm{N}(2)^{\prime}-\mathrm{Cd^{ \prime }}$ & $122.3(5)$ \\
\hline$C(11)-N(3)-C d$ & $122.2(4)$ & $C(11)^{\prime}-N(3)^{\prime}-C d^{\prime}$ & $124.5(4)$ \\
\hline$C(7)-N(3)-C d$ & $121.9(4)$ & $C(7)^{\prime}-N(3)^{\prime}-C d^{\prime}$ & $117.3(4)$ \\
\hline$C(6)^{\# 4}-N(1)-C(6)$ & $119.2(11)$ & $\mathrm{C}(6)^{\prime}-\mathrm{N}(1)^{\prime}-\mathrm{C}(6)^{\prime \# 5}$ & $115.7(11)$ \\
\hline$C(1)^{\# 4}-N(2)-C(1)$ & $119.3(9)$ & $\mathrm{C}(1)^{\prime}-\mathrm{N}(2)^{\prime}-\mathrm{C}(1)^{\prime \prime \prime 5}$ & $115.4(10)$ \\
\hline $\mathrm{C}(11)-N(3)-C(7)$ & $116.0(5)$ & $\mathrm{C}(7)^{\prime}-\mathrm{N}(3)^{\prime}-\mathrm{C}(11)^{\prime}$ & $118.0(5)$ \\
\hline$C(18)-N(4)-C(17)$ & $116.5(7)$ & $C(12)-N(5)-C(21)$ & $116.6(7)$ \\
\hline $\mathrm{C}(31)-\mathrm{N}(7)-\mathrm{C}(30)$ & $114.9(7)$ & & \\
\hline
\end{tabular}

Hydrogen bond distances $/ \AA$

$\begin{array}{llll}\mathrm{O}_{\mathrm{w}}-\mathrm{HO}_{\mathrm{wa}} \cdots \mathrm{O}(11) & 2.909(12) & \mathrm{O}_{\mathrm{w}}-\mathrm{HO}_{\mathrm{wb}} \cdots \mathrm{N}(7)^{*} & 2.776(12) \\ \mathrm{O}(1)-\mathrm{HO}_{1 \mathrm{a}} \cdots \mathrm{N}(5) & 2.722(10) & \mathrm{O}(1)-\mathrm{HO}_{1 \mathrm{~b}} \cdots \mathrm{O}_{\mathrm{w}} & 2.831(12) \\ \mathrm{O}(1)^{\prime}-\mathrm{HO}(1)^{\prime} \cdots \mathrm{O}_{\mathrm{w}} & 2.724(12) & \mathrm{O}(1)^{\prime}-\mathrm{HO}^{\prime \prime} \cdots \mathrm{O}(7)^{\#} & 2.825(10) \\ \mathrm{O}(4)-\mathrm{HO}(4) \cdots \mathrm{N}(4)^{*} & 2.693(9) & & \\ \text { Short distances/A } & & & \\ \mathrm{Cl}(1) \cdots \mathrm{H}(5)^{* *} & 3.199(13) & \mathrm{Cl}(1) \cdots \mathrm{H}(6)^{* *} & 3.206(13) \\ \mathrm{Cl}(1) \cdots \mathrm{H}(27)^{\# \#} & 3.153(13) & & \end{array}$

${ }^{\# 1} X, Y+1, Z ;{ }^{2}-X+1, Y,-Z+1 ;{ }^{3}-X+1, Y,-Z ;{ }^{* 4}-X$ $Y,-Z+1 ;{ }^{5}-X, Y,-Z ;{ }^{* 6} X, Y-1, Z_{i}^{*}{ }^{*} 1-X, Y, 1-Z$, ${ }^{\#}-X, Y,-Z ;{ }^{* * 1}-X, 1+Y, 1-Z ;{ }^{*} 1-X, Y, 1-Z$.

$\left[\mathrm{Cd}\left(4,4^{\prime}-\mathrm{bpy}\right)_{2}\left(\mathrm{H}_{2} \mathrm{O}\right)_{2}\right]^{2+}$ ion and $16.8(6)$ and $50.1(2)^{\circ}$ for the other $\left[\mathrm{Cd}^{\prime}\left(4,4^{\prime}-\mathrm{bpy}\right)_{2}\left(\mathrm{H}_{2} \mathrm{O}\right)_{2}\right]^{2+}$ ion. Coplanarity of pyridyl rings in coordinated 4,4'-bpy molecules is, however, not unusual for high dimensional square network polymers. For example, each $4,4^{\prime}$-bpy ligand in $\left[\mathrm{Zn}\left(4,4^{\prime} \text {-bpy }\right)_{2}\right] \mathrm{SiF}_{6} \cdot x \mathrm{DMF}$ is almost planar due to the existence of $\mathrm{C}-\mathrm{H} \cdots \mathrm{F}$ hydrogen bonds between $\alpha-\mathrm{C}-\mathrm{H}$ hydrogen atoms and fluorine atoms of the $\mathrm{SiF}_{6}{ }^{2-}$ ion, ${ }^{5}$ and half of the $4,4^{\prime}$-bpy ligands in $\left[\mathrm{Cd}\left(4,4^{\prime} \text {-bpy }\right)_{2}\left(\mathrm{NO}_{2}\right)_{2}\right] \cdot\left(\mathrm{C}_{6} \mathrm{H}_{4} \mathrm{Br}_{2}\right)_{2}$ are also planar owing to the host-guest interactions. ${ }^{7}$
The $\left[\mathrm{Cd}\left(4,4^{\prime} \text {-bpy }\right)_{2}\left(\mathrm{H}_{2} \mathrm{O}\right)_{2}\right]^{2+}$ square networks are stacked parallel to the $a b$ plane (Fig. 2). Uncoordinated 4,4'-bpy molecules are clathrated in square cavities, which are similar to those in $\left[\mathrm{Cd}\left(4,4^{\prime}-\mathrm{bpy}\right)_{2}\left(\mathrm{H}_{2} \mathrm{O}\right)_{2}\right]\left(\mathrm{PF}_{6}\right)_{2}$. $2\left(4,4^{\prime}\right.$-bpy $) \cdot 4 \mathrm{H}_{2} \mathrm{O}{ }^{1 \mathrm{a}}$ However, the 4-chloro-2-nitrophenol guest molecule is intercalated between $\left[\mathrm{Cd}\left(4,4^{\prime} \text {-bpy }\right)_{2}\left(\mathrm{H}_{2} \mathrm{O}\right)_{2}\right]^{2+}$ square networks with strong hydrogen bond between 4-chloro-2-nitrophenol and uncoordinated 4,4'-bipyridine [O4-HO4 $\cdots \mathrm{N}^{\#}$. 2.693 (3) A] rather than be clathrated into a square cavity as the uncoordinated $4,4^{\prime}$-bpy ligand does. Probably the chlorine atom in 4-chloro-2-ntrophenol plays a key role in incorporating this guest molecule since the short distances (about $3.2 \AA$ ) between $\mathrm{Cl} 1$ and $\mathrm{H} 5^{* *}, \mathrm{H}^{* *}$ attached to $\mathrm{C} 5^{* *}, \mathrm{C} 6^{* *}$ of the pyridine ring in $\left[\mathrm{Cd}\left(4,4^{\prime} \text {-bpy }\right)\left(\mathrm{H}_{2} \mathrm{O}\right)_{2}\right]^{2+}$ were observed, suggesting nonionic electrostatic attraction exists between 4-chloro-2nitrophenol guest molecule and $\left[\mathrm{Cd}\left(4,4^{\prime}-\text { bpy }\right)_{2}\left(\mathrm{H}_{2} \mathrm{O}\right)_{2}\right]^{2+}$ host as the short distance ( $3.2 \AA$ ) between $\mathrm{Br} 22$ and $\mathrm{H}^{\prime}$ ' of pyridine ring $\mathrm{B}^{\prime}$ implies nonionic electrostatic attraction between $\mathrm{C}_{6} \mathrm{H}_{4} \mathrm{Br}_{2}$ and $\left[\mathrm{Cd}\left(4,4^{\prime} \text {-bpy }\right)_{2}\left(\mathrm{NO}_{3}\right)_{2}\right]$ in $\left[\mathrm{Cd}\left(4,4^{\prime} \text {-bpy }\right)_{2}\left(\mathrm{NO}_{3}\right)_{2}\right] \cdot\left(\mathrm{C}_{6} \mathrm{H}_{4} \mathrm{Br}_{2}\right)_{2}{ }^{7}$ Furthermore, the crystal lattice is also stabilized by an extensive hydrogen bonding network between the coordinated and uncoordinated water molecules [2.831(12) and 2.724(12) $\AA$ for $\mathrm{O} 1-\mathrm{HO} 1 \mathrm{~b} \cdots \mathrm{O}_{\mathrm{w}}$ and $\left.\mathrm{O}^{\prime}-\mathrm{HO}^{\prime} \cdots \mathrm{O}_{\mathrm{w}}\right]$ as well as between the water molecules and the uncoordinated $4,4^{\prime}$ bpy $[2.722(10)$ and $2.776(12) \AA$ for $\mathrm{O} 1-\mathrm{HOla} \cdots \mathrm{N} 5$ and $\left.\mathrm{O}_{\mathrm{W}}-\mathrm{HO}_{\mathrm{W}} \cdots \mathrm{N} 7^{*}\right]$ and ionic perchlorate [2.909(12) and $2.825(10) \AA$ for $\mathrm{O}_{\mathrm{w}}-\mathrm{HO}_{\mathrm{w}} \mathrm{a} \cdots \mathrm{O} 11$ and $\left.\mathrm{O}^{\prime}-\mathrm{HO}^{\prime \prime} \cdots \mathrm{O}^{\#}\right]$.

Thermal analyses. The TDA curve of compound $\left[\mathrm{Cd}\left(4,4^{\prime}-\right.\right.$ bpy $)_{2}\left(\mathrm{H}_{2} \mathrm{O}_{2}\right]\left(\mathrm{ClO}_{4}\right)_{2} \cdot 1.5\left(4,4^{\prime}\right.$-bpy $) \cdot\left(\mathrm{C}_{6} \mathrm{H}_{4} \mathrm{NO}_{3} \mathrm{Cl}\right) \cdot$ $\mathrm{H}_{2} \mathrm{O}$ (Fig. 3) shows two endotherms between 30 and $150^{\circ} \mathrm{C}$. This is paralleled in the TGA plot with two weight losses of 1.7 and $3.4 \%$, respectively, corresponding to the losses of one uncoordinated and two coordinated water molecules. The third endotherm on the TDA curve is observed at $197.8^{\circ} \mathrm{C}$, with a weight loss of $15.9 \%$ as shown in the TGA plot, corresponding to the loss of a 4-chloro-2-nitrophenol molecule. The subsequent two endotherms on the TDA curve are observed at 250.4 and $290.4{ }^{\circ} \mathrm{C}$, which in the TGA curve show up as two weight losses of 14.5 and $14.3 \%$, respectively, corresponding to the loss of two 4,4'-bpy molecules step by step. Since $\left[\mathrm{Cd}\left(4,4^{\prime} \text {-bpy }\right)_{2}\left(\mathrm{H}_{2} \mathrm{O}\right)_{2}\right]\left(\mathrm{ClO}_{4}\right)_{2} \cdot 1.5\left(4,4^{\prime}\right.$-bpy $)$. $\left(\mathrm{C}_{6} \mathrm{H}_{4} \mathrm{NO}_{3} \mathrm{Cl}\right) \cdot \mathrm{H}_{2} \mathrm{O}$ and some decomposed products are potentially explosive, higher temperatures should be avoided for safety. In short, the thermal analyses of $\left[\mathrm{Cd}\left(4,4^{\prime} \text {-bpy }\right)_{2}\left(\mathrm{H}_{2} \mathrm{O}\right)_{2}\right]\left(\mathrm{ClO}_{4}\right)_{2} \cdot 1.5\left(4,4^{\prime}\right.$-bpy $) \cdot$ $\left(\mathrm{C}_{6} \mathrm{H}_{4} \mathrm{NO}_{3} \mathrm{Cl}\right) \cdot \mathrm{H}_{2} \mathrm{O}$ are in accordance with its structure.

Acknowledgments. This work was supported by a grant for a key research project from the State Science and the Technology Commission and the National Nature Science Foundation of China, as well as the Foundation 


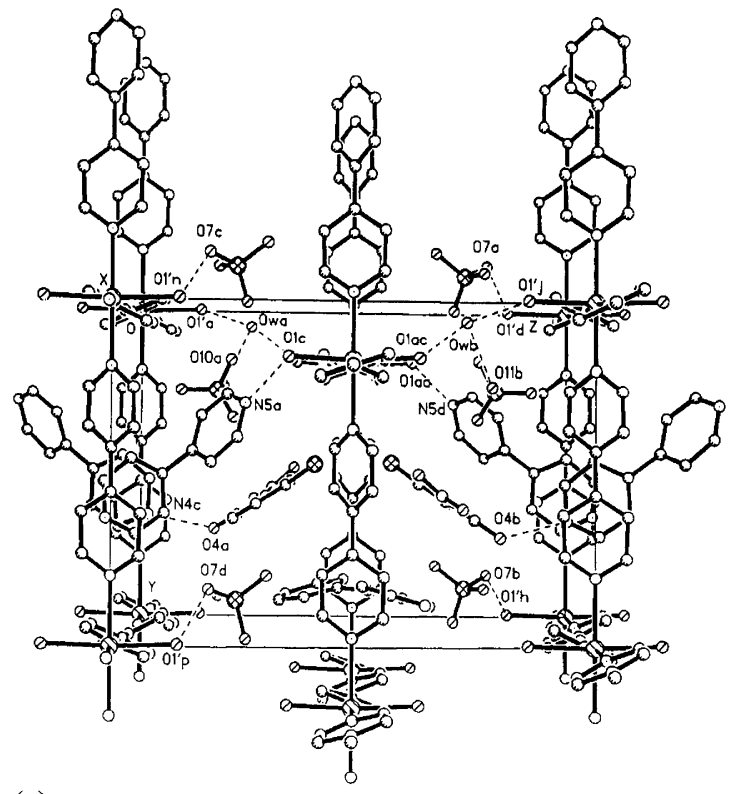

(a)

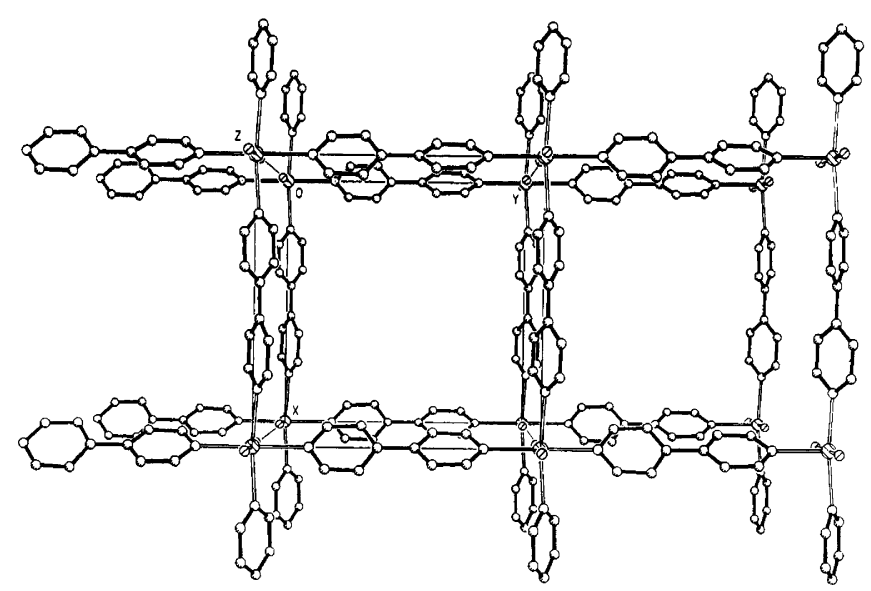

(b)

Fig. 2. (a) Projection down the $b$-axis of the title compound. (b) Projection down the $c$-axis of two square networks, the middle square network is omitted for clarity.

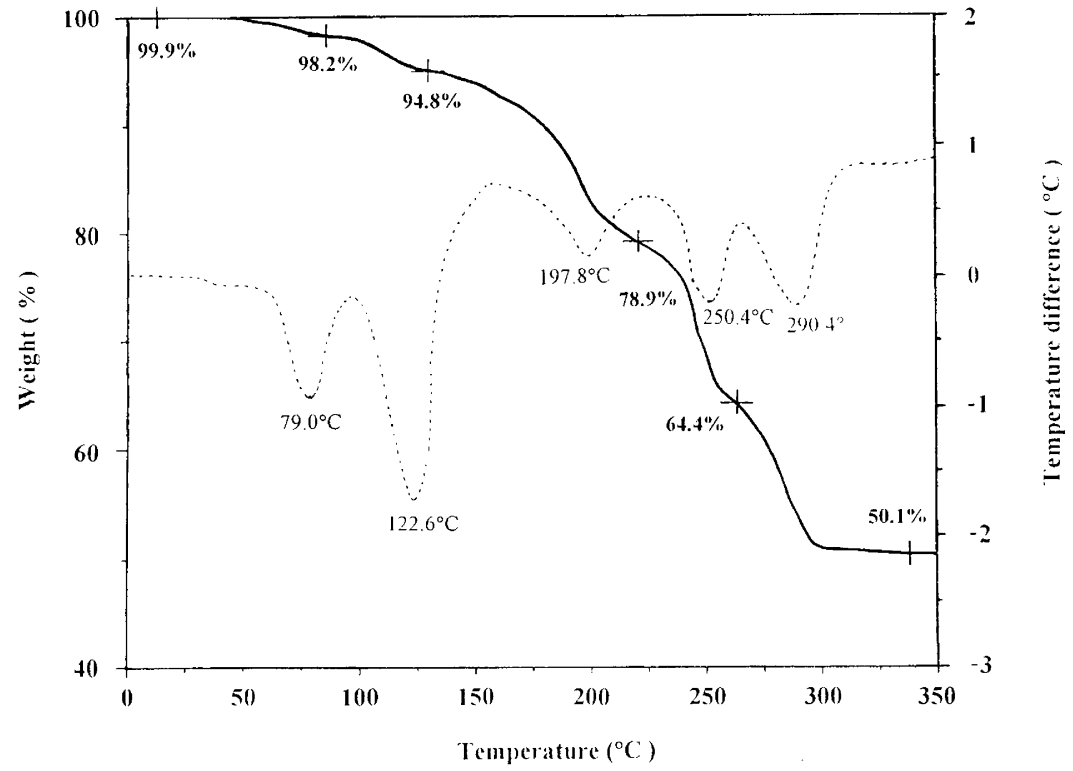

Fig. 3. The TGA/TDA curves of the title compound.

of the Chinese Postdoctoral Fellowship and the Fund of University of Malaya (IRPA Grant No. 09-02-03-0004).

\section{References}

1. See for example: (a) Robson, R., Abrahams, B. F., Batten, S. R., Gable, R. W., Hoskins, B. F. and Liu, J. Supramolecular Architecture (ACS Symp. Ser. 1992, Chap. 19, p. 449); (b) Zaworotko, M. Chem. Soc. Rev. (1994) 283; (c) Hoskins, B. F. and Robson, R. J. Am. Chem. Soc. 112 (1990) 1546; (d) Moore, J. S. and Lee, S. Chem. Ind. (1994) 283.
2. (a) Ermer, O. Adv. Mater. 3 (1991) 608; (b) Bowes, C. L. and Ozin, G. A. Adv. Mater. 8 (1996) 13; (c) Aumuller, A., Erk, P., Klebe, G., Hünig, S., von Schütz, J. U. and Werner, H.-P. Angew. Chem. Int. Ed. (1994) 283.

3. De Munno, G., Muñoz, M. C. and Julve, M. Inorg. Chem 30 (1991) 2701, and references therein.

4. Carlucci, L., Ciani, G., Proserpio, D. M. and Sironi, A. Angew. Chem., Int. Ed. Engl. 34 (1995) 1895.

5. Subramanian, S. and Zaworotko, M. J. Angew. Chem. Int. Ed. Engl. 34 (1995) 2127.

6. Gable, R. W., Hoskins, B. F. and Robson, R. J. Chem Soc. Chem. Commun. (1990) 1677, and reference therein.

7. Fujita, M., Kwon, Y. J., Washizu, S. and Ogura, K. J. Am. Chem. Soc. 116 (1994) 1151. 
LIU ET AL.

8. Kopfman, G. and Huler, R. Acta Crystallogr., Sect. B 24 (1968) 348; Shieldrick, G. M. Acta Crystallogr., Sect. A 46 (1990) 467

9. Shieldrick, G. M. SHELXL93, Program for Crystal Structure Refinement. University of Göttingen, Germany 1993.
10. Schaeffer, W. D., Dorsey, W. S., Skinner, D. A. and Christlan, C. G. J. Chem. Soc. 79 (1957) 5870.

Received December 30, 1997. 